\title{
Comparison of the atherosclerotic burden among asymptomatic patients vs matched volunteers
}

\author{
Alan Rozanski, MD, ${ }^{\mathrm{a}}$ Heidi Gransar, MS, ${ }^{\mathrm{b}, \mathrm{c}}$ Leslee Shaw, PhD, ${ }^{\mathrm{d}}$ \\ Nathan D. Wong, PhD, ${ }^{\text {e }}$ James Min, MD, ${ }^{\mathrm{f}}$ Romalisa Miranda-Peats, $M P H,{ }^{\text {b,c }}$ \\ Sean W. Hayes, $M D$, , c John D. Friedman, $M D, M P H,{ }^{b, c}$ \\ and Daniel S. Berman, MD, FACC ${ }^{\mathrm{b}, \mathrm{c}}$
}

Background. While asymptomatic patients should have a lower risk of cardiac events compared to symptomatic patients referred for cardiac stress testing, comparable event rates have been noted in some prior prognostic studies. To test if a high burden of undetected atherosclerosis among asymptomatic patients helps explain such findings, we compared atherosclerotic burden, as measured by coronary artery calcium (CAC) scanning, in propensitymatched groups of volunteers and asymptomatic patients.

Methods. CAC scans were performed on a research basis in 136 asymptomatic patients referred for exercise myocardial perfusion SPECT and in 1,398 volunteers. We performed matching by propensity scores to compare volunteers with the same CAD risk factor profile as our asymptomatic patients.

Results. Among our matched groups, asymptomatic patients had significantly greater mean CAC scores than volunteers $(394 \pm 805$ vs $151 \pm 349, P=.001)$, primarily due to a higher frequency of CAC scores $>1,000(15.4 \%$ vs $2.5 \%, P<.001)$. Inducible myocardial ischemia by SPECT was present in $7 \%$ of patients, but was selectively concentrated among those with CAC scores $>1,000$, occurring in $27.0 \%$ of such patients vs only $1.9 \%$ among patients with CAC scores $<1,000(P<.0001)$.

Conclusions. In contrast to asymptomatic volunteers, asymptomatic patients referred for cardiac stress testing possess more extensive atherosclerosis as measured by CAC. Among asymptomatic patients with high $\mathrm{CAC}$ scores, the frequency of concomitant inducible myocardial ischemia is high. These results help explain prior prognostic studies concerning asymptomatic patients and indicate the importance of making a clinical distinction between healthy subjects and asymptomatic patients with respect to atherosclerotic risk. ( $J$ Nucl Cardiol 2011;18:291-8.)

Key Words: Coronary calcification $\bullet$ ischemia $\cdot$ atherosclerosis $\cdot$ coronary artery disease

From the Division of Cardiology, ${ }^{\text {a }}$ St. Lukes Roosevelt Hospital, New York, NY; The Departments of Imaging and Medicine and the Burns and Allen Research Institute, ${ }^{\mathrm{b}}$ Cedars-Sinai Medical Center, Los Angeles, CA; Department of Medicine, David Geffen School of Medicine, ${ }^{\mathrm{c}}$ University of California, Los Angeles, CA; Department of Medicine, ${ }^{\mathrm{d}}$ Emory University School of Medicine, Atlanta, GA; The Heart Disease Prevention Program, ${ }^{\mathrm{e}}$ University of California, Irvine, CA; and Departments of Medicine and Radiology, ${ }^{\mathrm{f}}$ Weill Medical College of Cornell University and New York Presbyterian Hospital, New York, NY.

This study was supported by a grant from The Eisner Foundation, Los Angeles, CA. Funding was provided by the EISNER FOUNDATION, who had no role in the design or conduct of this study.

Received for publication Aug 6, 2010; final revision accepted Nov 20, 2010.

\section{INTRODUCTION}

Because approximately half of men and women experiencing sudden cardiac death do so without any prior clinical symptoms, ${ }^{1}$ how to best identify asymptomatic

Reprint requests: Daniel S. Berman, MD, FACC, The Departments of Imaging and Medicine and the Burns and Allen Research Institute, Cedars-Sinai Medical Center, 8700 Beverly Building, Room 1258, Los Angeles, CA 90048; bermand@cshs.org.

$1071-3581 / \$ 34.00$

Copyright $@ 2010$ The Author(s). This article is published with open access at Springerlink.com

doi:10.1007/s12350-010-9324-1 
patients who are at risk for future cardiac events is an important medical issue. A Bayesian approach for the identification of CAD risk was initially developed by Diamond and Forrester, ${ }^{2}$ based on patients' age, sex, chest pain symptoms, and consideration of patients' CAD risk factors. Because middle aged and older asymptomatic patients with multiple CAD risk factors may have an intermediate $(>15 \%)$ Bayesian likelihood of CAD, they are not uncommonly referred for cardiac stress testing. ${ }^{3}$ Patients presenting with chest pain symptoms have an even higher Bayesian likelihood of $\mathrm{CAD}$, which should thus place such patients at even higher risk for future cardiac events. ${ }^{4}$ Notably, however, comparisons of outcomes among patients with and without chest pain syndromes that have been referred for cardiac stress have revealed comparable event rates in some studies. ${ }^{5-7}$ Heretofore, an explanation for these findings has been lacking. One possibility is that asymptomatic patients referred for cardiac stress testing may have a greater than suspected burden of subclinical atherosclerosis that places such patients at increased risk for cardiac events. New technologies such as coronary artery calcium (CAC) scanning allow us to test this hypothesis. Accordingly, in this study, we compared the presence and magnitude of subclinical atherosclerosis, as measured by CAC scanning in a cohort of asymptomatic patients referred for cardiac stress testing to that measured in a group of matched asymptomatic volunteers.

\section{METHODS}

\section{Study Cohort}

The participants of this study included 136 asymptomatic patients (i.e., without chest pain symptoms), with a mean age of $59.2 \pm 10.0$, who were referred for exercise myocardial perfusion scintigraphy (MPS) at Cedars Sinai Medical Center (CSMC) by their referring physicians. These clinically referred patients underwent additional CAC scanning on a prospective research basis. Asymptomatic patients complaining of dyspnea were excluded, ${ }^{6}$ as were those with known CAD, cardiomyopathy, valvular disease, or those requiring pharmacologic stress testing. We separately evaluated a community cohort of 1,397 healthy asymptomatic volunteers who were recruited as part of a trial to assess the long-term impact of CAC scanning on subjects' health behaviors and risk profiles, the Early Identification of Subclinical Atherosclerosis by Noninvasive Imaging Research (EISNER) trial. We preferentially recruited males, 45-80 years, or females 55-80 years, with one or more CAD risk factors. These volunteers underwent CAC scanning but not MPS. This research was approved by the CSMC Institutional Review Board and all subjects signed informed consent.

\section{Clinical Information}

At the time of testing, all patients completed a questionnaire regarding demographic information, chest pain symptoms, cardiac risk factors, and medication use. A Bayesian pre-MPS likelihood of CAD was calculated for each patient based on the analysis of age, gender, risk factors, chest pain symptoms, and results of exercise electrocardiography according to a previously validated commercial program (CADENZA). ${ }^{8}$ Fasting blood samples were obtained to assess total cholesterol, HDL cholesterol, and triglycerides, with calculated LDL cholesterol values. Serum glucose levels were assessed by a Cholestech (Hayward, CA, USA) desktop chemical analyzer. Weight and height were obtained on each subject for calculation of body mass index (BMI). Using the clinical and serum measures, a Framingham 10-year risk of cardiac death or nonfatal myocardial infarction was calculated for each subject in accordance with published guidelines. ${ }^{9}$

\section{Stress Testing and Imaging Protocol}

Following symptom-limited Bruce protocol exercise, patients underwent separate-acquisition-gated dual isotope MPS as previously described, ${ }^{10}$ with thallium-201(3.0-4.5 mCi) injected at rest and technetium-99m sestamibi (25-40 mCi) injected at near maximal exercise. An elliptical $180^{\circ}$ acquisition and standard energy windows were used. Semi-quantitative visual interpretation was performed by experienced observers using a 5-point score for each of 20 myocardial segments and summed stress and rest scores were generated. Ischemia was defined by the presence of reversible defects in at least $5 \%$ of the myocardial segments. ${ }^{10}$

\section{Calcium Scanning}

Scanning was performed using either electron beam computed tomography (EBT) (Imatron C-150 or GE e-Speed) or multispiral computed tomography (MSCT) (Siemens Volume Zoom, Siemens Medical Systems). Each patient's scan consisted of approximately 30-40 slices of 3 or $2.5 \mathrm{~mm}$ for EBT and MSCT, respectively, with triggering at $50 \%-80 \%$ of the cardiac cycle. Foci of CAC were identified by an experienced technologist and scored using semi-automatic commercial software by detection of at least three contiguous pixels (voxel size $=1.03 \mathrm{~mm}^{3}$ ) of peak density $\geq 130$ Hounsfield units within a coronary artery. CAC scores were calculated according to the method of Agatston et al $^{11}$ Percentage CAC scores, based on age and gender, were assigned according to the database of Raggi et $\mathrm{al}^{12}$.

\section{Statistical Analysis}

Continuous variables were expressed as mean \pm standard deviation and were compared using two-sample $t$-tests for normal data and two-sample Wilcoxon rank-sum test for nonnormal data. Normality was assessed by using ShapiroFrancia, Shapiro-Wilk, and Skewness/Kurtosis tests for normality. Because the distribution of CAC scores are highly 
skewed, in addition to its identity form, a transformation toward normality in the form of $\log (\mathrm{CAC}+1)$ was also used (adding one to deal with the 0 scores). Categorical variables were compared using the Pearson $\chi^{2}$ test; the $\chi^{2}$ test for trend and Fisher Exact test for cell counts $<6$ were also used where appropriate. In order to adjust for differences in baseline characteristics between subjects and patients, all 136 were matched to the volunteer subjects on a 2:1 volunteer to patient ratio on the basis of age, gender, BMI, and history of the following coronary risk factors: high cholesterol, hypertension, smoking, diabetes, and family history of premature CAD. Matching was done using propensity scores that were derived from the predicted probabilities that resulted from a logistic regression model to predict being a patient vs being a subject. ${ }^{13}$ Since the matching was $2: 1$, the propensity matched groups were also compared as described above.

\section{RESULTS}

\section{Baseline Characteristics}

A comparison of the clinical characteristics among the two study groups is shown in Table 1 . The healthy volunteers and patients were comparable in age but there were a greater percentage of males among the patients. The patient group had a higher frequency of high cholesterol, hypertension, diabetes, and higher glucose levels. Serum cholesterol and LDL levels were lower but lipid-lowering usage was greater among the patient cohort. Overall, all but $11(8 \%)$ of our patient cohort had the presence of $\geq 1$ CAD risk factors and $96(71 \%)$ had $\geq 2$ CAD risk factors.

Table 1. Clinical characteristics in the study groups

\begin{tabular}{|c|c|c|c|}
\hline & $\begin{array}{c}\text { Healthy } \\
\text { volunteers } \\
(\mathbf{N}=\mathbf{1 , 3 9 8 )}\end{array}$ & $\begin{array}{c}\text { Asymptomatic } \\
\text { patients } \\
(\mathbf{N}=136)\end{array}$ & P-values \\
\hline Age & $58.6 \pm 8.6$ & $59.2 \pm 10.0$ & .41 \\
\hline Male & $739(52.9 \%)$ & $104(76.5 \%)$ & $<.001$ \\
\hline \multicolumn{4}{|l|}{ CAD risk factors } \\
\hline High cholesterol & 817 (58.4\%) & 100 (73.5\%) & .001 \\
\hline Hypertension & 583 (41.7\%) & 74 (54.4\%) & .004 \\
\hline Smoking & 91 (6.5\%) & $11(8.1 \%)$ & .48 \\
\hline Diabetes & $92(6.6 \%)$ & $22(16.2 \%)$ & $<.001$ \\
\hline Family history & $382(27.3 \%)$ & $35(25.7 \%)$ & .69 \\
\hline \# of CAD risk factors & $1.4 \pm 0.9$ & $1.7 \pm 1.0$ & $<.0001$ \\
\hline BMI & $27.7 \pm 5.3$ & $28.2 \pm 5.3$ & .09 \\
\hline Pre-test likelihood of CAD & $6.7 \pm 4.0$ & $7.9 \pm 4.7$ & .001 \\
\hline \multicolumn{4}{|l|}{ Medication use } \\
\hline Blood pressure medications & $450(32.2 \%)$ & $59(43.4 \%)$ & .008 \\
\hline Lipid lowering medications & $364(26.0 \%)$ & $56(41.2 \%)$ & $<.001$ \\
\hline \multicolumn{4}{|l|}{ Laboratory values } \\
\hline Glucose & $96.1 \pm 19.0$ & $101.2 \pm 29.4$ & .005 \\
\hline Cholesterol & $215.4 \pm 41.6$ & $203.0 \pm 47.6$ & .0001 \\
\hline LDL & $135.6 \pm 38.9$ & $124.1 \pm 41.7$ & .0002 \\
\hline $\mathrm{HDL}$ & $53.8 \pm 16.6$ & $51.5 \pm 16.2$ & .12 \\
\hline Triglycerides & $133.9 \pm 84.4$ & $135.7 \pm 104.3$ & .35 \\
\hline \multicolumn{4}{|l|}{ FRS } \\
\hline Mean score & $11.0 \pm 7.0$ & $13.0 \pm 8.2$ & .01 \\
\hline FRS $<\% 10$ & $618(56.0 \%)$ & $48(42.9 \%)$ & \\
\hline FRS $10 \%-20 \%$ & $319(28.9 \%)$ & $37(33.0 \%)$ & \\
\hline FRS $>20 \%$ & $167(15.1 \%)$ & $27(24.1 \%)$ & .01 \\
\hline \multicolumn{4}{|l|}{ Resting hemodynamic } \\
\hline Heart rate (bpm) & $66.3 \pm 10.3$ & $67.8 \pm 12.1$ & 0.22 \\
\hline Systolic BP (mmHg) & $133.1 \pm 17.5$ & $135.3 \pm 16.0$ & 0.06 \\
\hline Diastolic BP (mmHg) & $82.0 \pm 10.7$ & $80.0 \pm 8.0$ & 0.005 \\
\hline
\end{tabular}

$B P$, Blood pressure. 


\section{Comparison of CAC Scores in the Asymptomatic Groups}

Table 2 lists the CAC findings in the two study groups. The mean CAC score was more than twofold higher in the patient group. More volunteers than patients had normal CAC scans and conversely, there was a substantially greater frequency of patients with very high CAC scores (i.e., >1,000) compared to the volunteer group: $15.4 \%$ vs $2.9 \%, P<.001$. Similarly, the mean percentile CAC score was higher in the patient group and more patients than volunteers had a CAC percentile score $\geq 90 \%, P=.002$.

\section{Stress Test and Rest ECG Findings Among the Asymptomatic Patients}

Among the 136 patients, the mean exercise duration was $9.6 \pm 2.8 \mathrm{~min}$, with a mean peak heart rate of $155 \pm$ $13 \mathrm{bpm}$ and mean peak systolic/diastolic blood pressure of $173 / 77 \mathrm{mmHg}$. An ischemic SPECT study was noted in $9(7 \%)$ of the 136 patients, and in these patients, the mean CAC score was significantly higher than in the nonischemic SPECT patients $(1616 \pm 1182$ vs $307 \pm 700$, $P<.0001)$. An ischemic SPECT occurred in only 2 (1.9\%) of the patients with a CAC score $<1,000$ but in 7 $(27.0 \%)$ of the 26 patients with a CAC score $\geq 1,000$ $(P<.0001)$.

Forty-five (33\%) of the 136 patients had an abnormal ECG response during exercise testing, but of these 45, 42 (93\%) had a normal exercise SPECT study, and the mean CAC score was not significantly different among the 42 nonischemic SPECT patients with an abnormal exercise ECG response vs the 88 nonischemic
SPECT patients with a normal exercise ECG response $(355 \pm 581$ vs $275 \pm 755, P=.56)$.

We also repeated our analyses after excluding all patients manifesting an abnormal exercise ECG response. The substantial difference in mean CAC scores persisted in the remaining 91 asymptomatic patients compared to the healthy volunteers $(385 \pm 890$ vs $122 \pm 347, P<$ $.0001)$ as did the percent with CAC scores $>1,000(13.2 \%$ vs $2.5 \%, P<.0001)$.

Among the 136 referred patients, 40 (29\%) had an abnormal rest ECG as an indicated reason for testing. The mean CAC score in this group was also not higher compared to those patients who had a normal resting ECG $(279 \pm 734$ vs $413 \pm 829, P<.10)$. Other reasons for patient referral varied widely, including the referral of nine patients $(7 \%)$ for pre-operative evaluation.

\section{Comparison of the Volunteer and Patients Groups Following Propensity Analysis}

Following a 2:1 propensity matching of the volunteer and patient groups on the basis of age, gender, and each of the major CAD risk factors (hypercholesterolemia, hypertension, smoking, diabetes, family history, and $\mathrm{BMI}$ ), there were 272 propensity-matched volunteers and 136 propensity-matched patients. As shown in Table 3, these two groups manifested no significant differences in age, gender distribution, CAD risk factors, medication use, Framingham risk score (FRS) scores, or pre-test likelihood of CAD. Serum chemistries differed only slightly (mildly lower cholesterol, LDL, and triglyceride values in the patient cohort).

Table 2. Results of CAC scanning in the study groups

\begin{tabular}{llll}
\hline $\begin{array}{c}\text { Healthy } \\
\text { volunteers } \\
\mathbf{( N = ~ 1 , 3 9 8 )}\end{array}$ & $\begin{array}{c}\text { Healthy } \\
\text { volunteers } \\
\mathbf{( N = 1 , 3 9 8 )}\end{array}$ & $\begin{array}{c}\text { Asymptomatic } \\
\text { patients } \\
\mathbf{( N = 1 3 6 )}\end{array}$ & P-values \\
\hline Mean CAC score & $122 \pm 347$ & $394 \pm 805$ & $<.0001$ \\
Mean log CAC score & $2.2 \pm 2.4$ & $3.5 \pm 2.8$ & $<.0001$ \\
Distribution of CAC scores & $664(47.5 \%)$ & $42(30.9 \%)$ & \\
CAC $=0$ & $416(29.7 \%)$ & $37(27.2 \%)$ & \\
CAC 10-99 & $196(14.0 \%)$ & $25(18.4 \%)$ & $<.001$ \\
CAC 100-399 & $87(6.2 \%)$ & $11(8.1 \%)$ & $<.0001$ \\
CAC 400-999 & $35(2.5 \%)$ & $21(15.4 \%)$ & \\
CAC $\geq 1000$ & $33.2 \pm 35.8$ & $47.3 \pm 37.9$ & \\
CAC percentile & $873(62.5 \%)$ & $66(48.5 \%)$ & $<.001$ \\
CAC $<$ 50th percentile & $257(18.4 \%)$ & $25(18.4 \%)$ & \\
CAC 50-74th percentiles & $160(11.4 \%)$ & $19(14.0 \%)$ & \\
CAC 75-89th percentiles & $108(7.7 \%)$ & $26(19.1 \%)$ & \\
CAC $\geq$ 90th percentile & & & \\
\hline
\end{tabular}


Table 3. Clinical characteristics of the propensity-matched study groups

\begin{tabular}{|c|c|c|c|}
\hline & $\begin{array}{l}\text { Healthy volunteers } \\
(\mathbf{N}=\mathbf{2 7 2})\end{array}$ & $\begin{array}{c}\text { Asymptomatic } \\
\text { patients }(N=136)\end{array}$ & P-values \\
\hline Age $^{a}$ & $58.5 \pm 9.1$ & $59.2 \pm 10.0$ & .43 \\
\hline Male $^{\mathrm{a}}$ & $207(76.1 \%)$ & $104(76.5 \%)$ & .93 \\
\hline \multicolumn{4}{|l|}{ CAD risk factors } \\
\hline High cholesterol $^{\mathrm{a}}$ & $198(72.8 \%)$ & $100(73.5 \%)$ & .88 \\
\hline Hypertension $^{\mathrm{a}}$ & $168(61.8 \%)$ & $74(54.4 \%)$ & .15 \\
\hline Smoking ${ }^{\mathrm{a}}$ & $14(5.2 \%)$ & $11(8.1 \%)$ & .24 \\
\hline Diabetes $^{\mathrm{a}}$ & $40(14.7 \%)$ & $22(16.2 \%)$ & .70 \\
\hline Family history ${ }^{a}$ & $64(23.5 \%)$ & $35(25.7 \%)$ & .62 \\
\hline \# of CAD factors & $1.8 \pm 0.9$ & $1.7 \pm 1.0$ & .82 \\
\hline $\mathrm{BMI}^{\mathrm{a}}$ & $28.4 \pm 5.1$ & $28.2 \pm 5.3$ & .65 \\
\hline \multicolumn{4}{|l|}{ Medication use } \\
\hline Blood pressure medications & $123(45.2 \%)$ & $59(43.4 \%)$ & .73 \\
\hline Lipid lowering medications & $99(36.4 \%)$ & $56(41.2 \%)$ & .35 \\
\hline \multicolumn{4}{|l|}{ Laboratory values } \\
\hline Glucose & $100.6 \pm 22.4$ & $101.2 \pm 29.4$ & .67 \\
\hline Cholesterol & $211.5 \pm 43.6$ & $203.0 \pm 47.6$ & .03 \\
\hline LDL & $133.6 \pm 39.5$ & $124.1 \pm 41.7$ & .01 \\
\hline $\mathrm{HDL}$ & $50.4 \pm 16.5$ & $51.5 \pm 16.2$ & .32 \\
\hline Triglycerides & $140.9 \pm 87.2$ & $135.7 \pm 104.3$ & .05 \\
\hline \multicolumn{4}{|l|}{ FRS } \\
\hline Mean score & $13.9 \pm 8.3$ & $13.0 \pm 8.2$ & .35 \\
\hline FRS $<10 \%$ & $89(42.8 \%)$ & $48(42.9 \%)$ & \\
\hline FRS $10 \%-20 \%$ & $61(29.3 \%)$ & $37(33.0 \%)$ & \\
\hline FRS $>20 \%$ & $58(27.9 \%)$ & $27(24.1 \%)$ & .70 \\
\hline \multicolumn{4}{|l|}{ Resting hemodynamics } \\
\hline Heart rate (bpm) & $66.5 \pm 10.6$ & $67.8 \pm 12.1$ & .39 \\
\hline Systolic BP (mmHg) & $137.1 \pm 17.2$ & $135.3 \pm 16.0$ & .50 \\
\hline Diastolic BP (mmHg) & $85.8 \pm 11.2$ & $80.0 \pm 8.0$ & $<.0001$ \\
\hline
\end{tabular}

a Propensity-matched on these clinical risk factors.

Despite this propensity matching, the mean CAC score remained significantly higher in the patient group compared to the healthy volunteers (Table 4). This difference in mean CAC scores within the propensity-matched subgroups was again due to a significantly greater percentage of patients with CAC scores $>1,000$ compared to propensity-matched healthy volunteers (Figure 1).

\section{DISCUSSION}

In this study, asymptomatic patients who were routinely referred for stress-rest myocardial perfusion SPECT were compared to a large group of asymptomatic individuals, recruited on a research basis as part of the EISNER study. In neither group was coronary calcium scanning used to determine the selection of subjects for study. Despite the absence of chest pain in both groups, the asymptomatic patients had a substantially greater mean CAC score, reflecting greater underlying atherosclerosis. This difference was due to a substantially greater concentration of patients with high CAC scores among the patient group. Nearly one-fourth of the asymptomatic patients had CAC scores $>400$ and $\sim 15 \%$ had CAC scores $>1,000$. By contrast, $<3 \%$ of comparably aged healthy volunteers had CAC scores $>1,000$.

Since the asymptomatic stress test patients were older, contained more males, and had a substantially greater frequency of CAD risk factors, we performed propensity matching to define a subgroup of volunteers and patients with the same risk factor profile. Because of our large volunteer population, we were able to perform this matching without excluding any patients. Among the propensity-matched subgroups, the greater frequency of patients with severe CAC scores $(>1,000)$ persisted among the patients compared to their matched controls. 
Table 4. Results of CAC scanning in the propensity-matched study groups

\begin{tabular}{llll}
\hline & $\begin{array}{c}\text { Healthy } \\
\text { volunteers } \\
(\mathbf{N}=\mathbf{2 7 2})\end{array}$ & $\begin{array}{c}\text { Asymptomatic } \\
\text { patients } \\
(\mathbf{N}=\mathbf{1 3 6})\end{array}$ & P-values \\
\hline $\begin{array}{l}\text { Mean CAC score } \\
\text { Distribution of CAC scores }\end{array}$ & $152 \pm 349$ & $394 \pm 805$ & .001 \\
CAC = & $111(40.8 \%)$ & $42(30.9 \%)$ & \\
CAC 1-9 & $24(8.8 \%)$ & $9(6.6 \%)$ & \\
CAC 10-99 & $63(23.2 \%)$ & $28(20.6 \%)$ & \\
CAC 100-399 & $34(12.5 \%)$ & $25(18.4 \%)$ & $<.001$ \\
CAC 400-999 & $32(11.8 \%)$ & $11(8.1 \%)$ & .002 \\
CAC $\geq 1000$ & $8(2.9 \%)$ & $21(15.4 \%)$ & \\
CAC percentile & $35.6 \pm 35.4$ & $47.3 \pm 37.9$ & \\
CAC $<$ 50th percentile & $165(60.7 \%)$ & $66(48.5 \%)$ & .008 \\
CAC 50-74th percentiles & $55(20.2 \%)$ & $25(18.4 \%)$ & \\
CAC 75-89th percentile & $29(10.7 \%)$ & $19(14.0 \%)$ & \\
CAC $\geq$ 90th percentile & $23(8.5 \%)$ & $26(19.1 \%)$ & \\
\hline
\end{tabular}

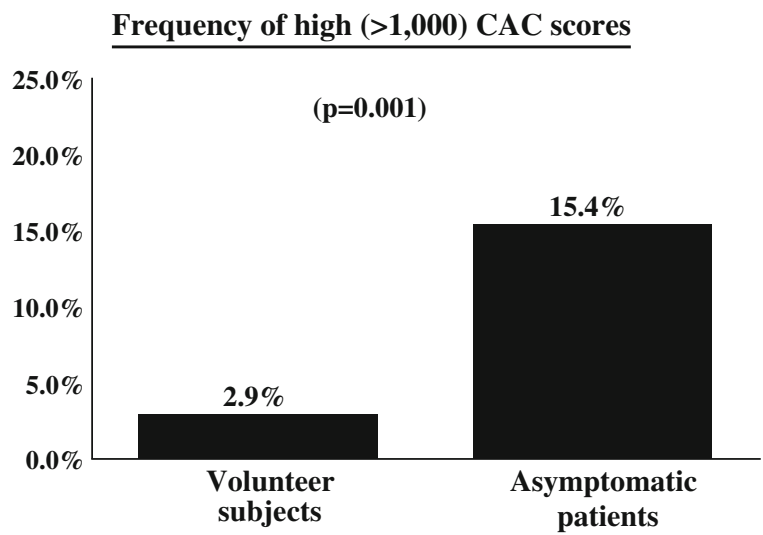

Figure 1. Frequency of CAC scores $>1,000$ ( $y$-axis) among the 272 propensity-matched asymptomatic volunteers and 136 asymptomatic patients. A substantially greater frequency of CAC scores $>1,000$ was noted within the patient group.

Besides the presence of cardiac risk factors, patients are commonly referred for exercise SPECT because of an ischemic-appearing ECG response during treadmill exercise. In our study, approximately one-third of our asymptomatic patients had such abnormal responses. However, $93 \%$ of these abnormal ECG responses were associated with a normal exercise SPECT study, indicating that they probably represented false positive response for inducible myocardial ischemia in our patient population. We found that the magnitude of CAC was not significantly different among the nonischemic SPECT subgroups that manifested a normal vs abnormal ECG response during exercise testing. Moreover, excluding all patients with a positive exercise ECG response to exercise did not change the results of our study. Thus, other factors must be operative to explain our findings.

\section{Potential Explanations for Increased Atherosclerosis in Asymptomatic Patients}

Various mechanisms may have potentially contributed to the increased atherosclerosis among our asymptomatic patients. Potentially, our patients could have had increased severity of CAD risk factors, including a more severe family history of premature CAD. Alternatively, the chronicity of CAD risk factors could have been greater within our patient population. These potentially important data were not obtained in our study. In addition, other CAD factors not analyzed in our data could have served as intangible causes for increased cardiac risk, such as increase in $\mathrm{Lp}(\mathrm{a})$, small dense LDL, or homocysteine measurements. In addition, various noncoronary disease processes that help promote more fatigue, tension, and other somatic complaints that lead to physician visits could be preferentially operative in patient populations, including a higher frequency of inflammatory diseases such as arthritis and autoimmune disease, ${ }^{14,15}$ and more chronic stress or depression. These common conditions have been linked to various pathophysiological mechanisms that may interact synergistically with conventional CAD risk factors to promote accelerated atherosclerosis in patients. ${ }^{16,17}$ Prospective study is needed to assess these and other potential explanations for our findings. 


\section{Relevance to Prior Clinical Outcome Studies in Stress Test Populations}

Among patients studied by planar thallium imaging in the 1980s, ${ }^{5}$ and then subsequently with SPECT imaging in the $1990 \mathrm{~s},{ }^{6}$ we noted that asymptomatic patients had cardiac event rates which were comparable to patients with chest pain symptoms. Christopher Jones et $\mathrm{al}^{7}$ have noted similar findings among their stress test patients. In other study, we have observed that asymptomatic patients have a frequency of inducible myocardial ischemia that is comparable to that observed among symptomatic patients referred for stress testing. ${ }^{10}$ Our current findings suggest that a potential reason for these prior observations is the existence of clinically silent but substantial atherosclerotic burden among some asymptomatic patients. The higher risk of this subgroup is further suggested by our observation of a high rate of inducible myocardial ischemia in over one-fourth of our patients with CAC scores $>1,000$. Of note, in prior studies involving a mixed cohort of patients with and without chest pain symptoms, a CAC score $>400$ has been noted to serve as a threshold for observing increased myocardial ischemia, ${ }^{18,19}$ but pending further study, perhaps the CAC score threshold for ischemia may be higher in asymptomatic patients compared to those with chest pain symptoms.

\section{Limitations}

Since our patient population was predominantly male, the role of gender could not be assessed in our study, nor did we assess the role of socioeconomic status or psychosocial risk factors. In addition, as aforementioned, knowledge concerning the temporal duration as well as the intensity of CAD risk factors may have been relevant to our results and would be useful parameters to assess in prospective studies comparing the presence and magnitude of coronary risk factors to measurements of atherosclerosis.

\section{Clinical Relevance}

Multiple potential means exist for screening asymptomatic patients, including the use of global algorithms such as the FRS, Bayesian analysis of CAD likelihood, the use of cardiac stress testing, and more recently, the application of CAC scanning. Recent study has defined limitations of relying on the FRS score to screen for underlying $\mathrm{CAD}^{20}$ and in our study there was no difference between FRS in our matched volunteers and asymptomatic patients, despite the presence of more atherosclerosis in the latter group. Similarly, a limitation to Bayesian analysis is that it was developed for the prediction of angiographically significant CAD (i.e.,
$>50 \%$ cross-luminal narrowing) and thus may not be a good predictor of atherosclerosis, per se. Indeed, the Bayesian likelihood of CAD was also comparable in our matched volunteers and patients. Also pointing to the limitation of Bayesian analysis for screening purposes is recent data indicating a poor correlation between Bayesian estimates of CAD likelihood and measurement of CAC scores. ${ }^{21}$ In addition, our study offers fresh insight regarding the limitation of using stress testing to screen for underlying disease among asymptomatic patients. That is, the application of SPECT imaging in our patients yielded only $7 \%$ with ischemic responses, compared to $70 \%$ with evidence of atherosclerosis by CAC scanning, representing a wide differential of individuals identified at risk. However, since the induction of inducible myocardial ischemia has been shown to be uncommon among patients with CAC scores $<400,{ }^{18,19}$ an approach that first uses CAC scanning might serve as a useful means of both narrowing and improving the selection of asymptomatic patients for subsequent cardiac stress testing. While the cost-effectiveness of such an approach needs to be prospectively evaluated, a recent study has suggested that the application of CAC scanning has the potential to reduce health costs by virtue of selectively concentrating downstream medical testing among asymptomatic subjects with higher CAC scores. ${ }^{22}$

\section{CONCLUSIONS}

Compared to asymptomatic volunteers, asymptomatic patients with comparably matched CAD risk factor profiles contain substantially more individuals with severe, clinically undetected atherosclerosis, as evidenced by CAC scores $>1,000$. This observation may help explain the results of outcome studies that have found overlapping cardiac event rates among asymptomatic vs symptomatic patients referred for cardiac stress testing. Prospective study is warranted to test the use of CAC scanning as a cost-effective triaging tool for the work-up of asymptomatic patients.

\section{Acknowledgments}

Dr Berman has grants from Lantheus Medical Imaging, Inc., and is on the speaker bureau for Astellas Pharma, Inc. The other authors have reported that they have no conflicts of interest.

\section{Open Access}

This article is distributed under the terms of the Creative Commons Attribution Noncommercial License which permits any noncommercial use, distribution, and reproduction in any medium, provided the original author(s) and source are credited. 


\section{References}

1. Rosamond W, Flegal K, Friday G, et al. Heart disease and stroke statistics-2007 update: A report from the American Heart Association Statistics Committee and Stroke Statistics Subcommittee. Circulation 2007;115:e69-71.

2. Diamond GA, Forrester J. Analysis of probability as an aid in the clinical diagnosis of coronary-artery disease. N Engl J Med 1979;300:1350-8.

3. Diamond GA, Denton TA, Berman DS, Cohen I. Prior restraint: A Bayesian perspective on the optimization of technology utilization for diagnosis of coronary artery disease. Am J Cardiol 1995;76:82-6.

4. Diamond GA, Staniloff HM, Forrester JS, et al. Computer-assisted diagnosis in the noninvasive evaluation of patients with suspected coronary artery disease. J Am Coll Cardiol 1983;1:444-55.

5. Staniloff HM, Forrester JS, Berman DS, Swan HJC. Prediction of death, myocardial infarction, and worsening chest pain using thallium scintigraphy and exercise electrocardiography. J Nucl Med 1986;27:1842-8.

6. Abidov A, Rozanski A, Hachamovitch R, et al. Prognostic significance of dyspnea in patients referred for cardiac stress testing. N Engl J Med 2005;353:1889-98.

7. Christopher Jones R, Pothier CE, Black-stone EH, Lauer MS. Prognostic importance of presenting symptoms in patients undergoing exercise testing for evaluation of known or suspected coronary disease. Am J Med 2004;117:380-9.

8. Diamond GA. Computer-assisted diagnosis and evaluation of coronary artery disease (version 5.04.3). Bainbridge Island, WA: Advanced Heuristics; 2000.

9. Wilson PWF, D'Agostino RB, Levy D, Belanger AM, Silbershatz H, Kannel WB. Prediction of coronary heart disease using risk factor categories. Circulation 1998;97:1837-47.

10. Berman DS, Hachamovitch R, Kiat H, et al. Incremental value of prognostic testing in patients with known or suspected ischemic heart disease: A basis for optimal utilization of exercise technetium-99m Sestamibi myocardial perfusion single-photon emission computed tomography. J Am Coll Cardiol 1995;26:639-47.
11. Agatston AS, Janowitz WR, Hildner FJ, et al. Quantification of coronary artery calcium using ultrafast computed tomography. J Am Coll Cardiol 1990;15:827-32.

12. Raggi P, Cooil B, Callister TQ. Use of electron beam tomography date to develop models for the prediction of hard coronary events. Am Heart J 2001;141:375-82.

13. Rosenbaum PR, Rubin DB. Reducing bias in observational studies using subclassification on the propensity score. J Am Stat Assoc 1984;79:516-24.

14. Chung CP, Oeser A, Raggi P, et al. Increases in coronary artery atherosclerosis in rheumatoid arthritis: Relationship to disease duration and cardiovascular risk factors. Arthritis Rheum 2005;52: 3045-53.

15. Gelfand JM, Neimann AL, Shin DB, Wang X, Margolis DJ, Troxel AB. Risk of myocardial infarction in patients with psoriasis. JAMA 2006;296:1735-41.

16. Rozanski A, Blumenthal JA, Davidson KW, Saab PG, Kubzansky L. The epidemiology, pathophysiology, and management of psychosocial risk factors in cardiac practice: The emerging field of behavioral cardiology. J Am Coll Cardiol 2005;45:637-51.

17. Tracy RP. Emerging relationships of inflammation, cardiovascular disease and chronic diseases of aging. Int J Obesity 2003;27:S29-34.

18. He ZX, Hedrick TD, Pratt CM, et al. Severity of coronary artery calcification by electron beam computed tomography predicts silent myocardial ischemia. Circulation 2000;101:244-51.

19. Berman DS, Wong ND, Gransar H, et al. Relationship between stressinduced myocardial ischemia and atherosclerosis measured by coronary calcium tomography. J Am Coll Cardiol 2004;44:923-30.

20. Polonsky TS, McClelland RL, Jorgensen NW, et al. Coronary artery calcium score and risk classification for coronary heart disease prediction. JAMA 2010;303:1610-6.

21. Rozanski A, Gransar H, Wong ND, et al. Use of coronary calcium scanning for predicting inducible myocardial ischemia: Influence of patients' clinical presentation. J Nucl Cardiol 2007;14:669-79.

22. Shaw LS, Min JK, Budoff M, Gransar H, Rozanski A, Hayes SW, Friedman JD, Miranda R, Wong ND, Friedman JD. Induced cardiovascular procedural costs and resource consumption patterns after coronary artery calcium scanning. J Am Coll Cardiol 2009;54: 1258-67. 